\title{
The Use of Finite Loading to Guide Short-Term Capacity Adjustments in Make-To-Order Job Shops: An Assessment by Simulation
}

Matthias Thürer (corresponding author) and Mark Stevenson

\author{
Name: $\quad$ Prof. Matthias Thürer \\ Institution: Jinan University \\ Address: $\quad$ Institute of Physical Internet \\ School of Electrical and Information Engineering \\ Jinan University (Zhuhai Campus) \\ 519070, Zhuhai, PR China \\ E-mail: matthiasthurer@workloadcontrol.com \\ Name: $\quad$ Prof. Mark Stevenson \\ Institution: Lancaster University \\ Address: $\quad$ Department of Management Science \\ Lancaster University Management School \\ Lancaster University \\ LA1 4YX - U.K. \\ E-mail: $\quad$ m.stevenson@lancaster.ac.uk
}

Keywords: Capacity Planning; Workload Control; Job Shop; Make-To-Order Production; Finite Loading. 


\title{
The Use of Finite Loading to Guide Short-Term Capacity Adjustments in Make-To-Order Job Shops: An Assessment by Simulation
}

\begin{abstract}
Although there is a broad literature on capacity management, there has been only limited attention on how to support short-term capacity control decisions, especially in high-variety make-to-order shops. While finite loading has been identified as a potential means of guiding capacity adjustments, the actual performance impact of this solution has not been adequately assessed. Using a simulation model of a make-to-order job shop, we compare the performance impact of: (i) four different forward and backward finite loading methods that guide adjustments; and, (ii) a method recently presented in the literature that uses a load threshold to trigger when and where to adjust capacity. Results confirm the potential of finite loading to improve performance when compared to a general capacity increase. Yet all four methods are outperformed by the load trigger method. The capacity adjustments made under finite loading methods are determined by individual jobs and their properties. This may lead to no adjustments despite an overload period (e.g. if a job has a long due date but only one overload station in its routing) or to unnecessary adjustments when there is no overload (e.g. if a large job has a tight due date). This finding draws into question the use of finite loading altogether and reinforces the importance of the load trigger method.
\end{abstract}

Keywords: Capacity Planning; Workload Control; Job Shop; Make-To-Order Production; Finite Loading. 


\section{Introduction}

Capacity management is an important production control function that significantly influences firm performance. It is often divided into different time horizons and stages in manufacturing firms, ranging from long-term capacity planning through to short-term capacity control (Wortmann et al., 1996; Olhager et al., 2011). More recently, Tenhiälä (2011) divided capacity management into rough-cut capacity planning, capacity requirements planning, and finite loading. Finite loading precedes the actual execution of capacity management decisions and exercises capacity control, i.e. it determines when and where short-term capacity adjustments such as overtime, additional shifts, or reduced working hours are required (Lödding, 2012). In this study, we use simulation to explore the performance impact of four different forward and backward finite loading methodologies in a make-toorder job shop and compare their performance with that of a load trigger method recently presented in the literature (Land et al., 2015).

Most capacity management research to date has focused on long-term or medium-term capacity decisions. Mathematical modelling has dominated this literature, arguably because this approach is able to optimize the use of capacity when presented with a fixed set of jobs for a given period of time; see, for example, Martinez-Costa et al. (2014) for a recent review on mathematical programming models in this context. This deterministic context is also present in most studies on stochastic capacity planning. (e.g. Chen et al. 2002; Hood et al., 2003; Geng et al., 2009; Lin et al., 2014), where the stochastic element is the demand in the time period. But this demand is assumed to be certain, i.e. it is known or given beforehand. When demand is uncertain, this is typically modelled by a set of scenarios with different probabilities of occurrence, which are then evaluated to create a robust solution (Geng \& Jiang, 2009). Similarly, studies on production scheduling that consider finite capacity - e.g. in the context of Material Requirements Planning (MRP; e.g. Chen \& Ji, 2007; Rossi et al., 2017) - rely on a given demand for which the schedule is then optimized.

From the above, it follows that the assumption in most studies on capacity management is that demand is deterministic; but this assumption is violated in high-variety make-to-order shops, where demand may arrive at any moment in time. Thus, make-to-order shops need to be able to evaluate, at any moment in time, if capacity needs to be adjusted and subsequently when and where it should be adjusted. In this context, short-term capacity decisions play a key role. Further, in such a context it is argued that optimization algorithms do not present a feasible solution, since: (i) the optimization would need to be executed each time a new job 
arrives; and, (ii) a previously optimal solution may turn out to be far from optimal when a new job arrives and needs to be incorporated into the existing schedule.

One method specifically designed to accomplish the challenging task of guiding short-term capacity decisions in a make-to-order context is finite loading, including both forward and backward finite loading methods. These finite loading methods are an essential part of workload controlling methods that build upon input/output control (I/OC, from Plossl \& Wight 1971), such as the Workload Control concept developed in the UK at Lancaster University (e.g. Kingsman et al., 1989; Hendry \& Kingsman, 1993) and Load Oriented Manufacturing Control (LOMC) developed at Hanover University in Germany (e.g. Bechte, 1994; Wiendahl, 1995). However, although this body of Workload Control literature has theoretically developed this method and advocated its potential use for capacity (or output) control, the actual performance impact of capacity adjustments based on finite loading in make-to-order shops has, to the best of our knowledge, not been assessed. A few simulation studies have assessed the performance impact of capacity adjustments (e.g. Hendry et al., 1989; Kingsman \& Hendry, 2002), but these studies only considered the capacity of the system as a whole system. They did not consider the capacity of individual stations, which is key to understanding where adjustments should be made. In response, we use simulation to explore the performance impact of different forward and backward finite loading methods and compare their performance with that of a workload trigger method recently presented in the literature (Land et al., 2015). The objective is to provide guidance to managers of maketo-order shops concerning which method to apply in practice to support capacity control decisions.

The remainder of this paper is structured as follows. In Section 2, we review theory on finite loading to provide the background to our study. The simulation model used to evaluate the performance of the different capacity control methods is then described in Section 3 before the results are presented, discussed, and analysed in Section 4. Finally, conclusions are provided in Section 5.

\section{Background}

This study focuses on finite loading and how it can be used to determine if, when, and where short-term capacity adjustments should take place. It does not focus on the actual execution of capacity management decisions, nor does it seek to answer the question of how capacity adjustments eventually should and/or can be realized, e.g. through overtime or additional shifts. Capacity control through finite loading is considered to precede this question. Section 
2.1 first discusses the use of finite loading in make-to-order contexts to identify the finite loading methods that should be considered in our study. Our research motivation and questions are then outlined in Section 2.2.

\subsection{Finite Loading Mechanisms in Make-to-Order Contexts}

Finite loading has many uses within production control. It has been used to determine short yet feasible due dates (e.g. Bertrand, 1983a, 1983b; Moses et al., 2004; Thürer et al., 2013), to decide on the acceptance of orders (e.g. Corti et al. 2006), to decide on the sub-contracting of orders (e.g. Thürer et al., 2014), and to guide the decision concerning when an order should be released to the shop floor (e.g. Ragatz \& Mabert, 1988; Bobrowski, 1989; Ahmed \& Fisher, 1992; Kim \& Bobrowski, 1995; Cigolini et al., 1998), Yet although finite loading mechanisms have also been advocated as means to guide short-term capacity adjustments, their actual usage has seldom been reported in the literature. The main exceptions are Hendry et al. (1989) and Kingsman \& Hendry (2002), but these two studies focused on the capacity of the whole system (instead of scheduling capacity for each individual station).

\subsubsection{Forward and Backward Finite Loading Methods from the Literature}

Both forward and backward finite scheduling determine an allowance for operation throughput times by fitting the workload to the available capacity. Take $R_{j}$ to be the ordered set of operations $\left(i, \ldots n_{j}\right)$ in the routing of job $j$. For forward scheduling, operation due dates $d_{i j}$ for each operation $i$ in $R_{j}$ are calculated starting from the arrival date. Under finite loading, a dynamic factor $F_{i j}\left(W_{s t}, C_{s t}\right)$ dependent on both the workload $\left(W_{s t}\right)$ and timephased capacity $\left(C_{s t}\right)$ at time $t$ at station $s$ performing operation $i$ of job $j$ is added to the operation due date that would otherwise be determined by assuming infinite capacity (see Equation (1)).

$d_{i j}=d_{i-1 j}+p_{i j}+a+F_{i j}\left(W_{s t}, C_{s t}\right)$

\footnotetext{
$p_{i j} \quad$ processing time of operation $i$

a minimum allowance for the operation throughput time
}

For backward loading, a similar procedure is applied but operation start dates $r_{i j}$ for each operation in the routing of a job are scheduled, where $r_{n+1 j}$ is equal to the due date and $n$ is the number of operations in the routing of the job (see Equation (2)). 
$r_{i j}=r_{i+1 j}-p_{i j}-a-F_{i j}\left(W_{s t}, C_{s t}\right)$

There are different ways to calculate $F_{i j}\left(W_{s t}, C_{s t}\right)$ where the main differences concern how workload and capacity are calculated. Arguably the simplest way is subdividing the future time horizon into time buckets $t$ and calculating a straightforward measure for each time bucket. This is known as Forward Finite Loading (FFL) and Backward Finite Loading (BFL). Only the FFL procedure is described here as the procedure for BFL is the same but in reverse, i.e. scheduling takes place from the due date backwards.

- If $d_{i j}$ calculated assuming infinite capacity (i.e. $F_{i j}\left(W_{s t}, C_{s t}\right)$ is zero) falls in a time bucket where the station to perform operation $i$ has sufficient free capacity to include the workload contribution of the job $p_{i j}$, that is $W_{s t}+p_{i j} \leq N_{s t}$ with $N_{s t}$ representing the capacity norm of the time bucket, then the operation is successfully scheduled into the time bucket.

- If no or insufficient capacity is available, the next time bucket is considered until a time bucket is reached in which the (full) workload contribution can be successfully loaded.

The above procedure is repeated for the next operation in the routing of a job until all operation due dates have been determined, with the last operation due date becoming the due date of the order. This forward loading procedure is efficient since: a new job's workload is inserted into the schedule without adjusting previously scheduled orders (Moses et al., 2004); and, the search direction is restricted so that only $t$ capacity checks need to be performed (Bertrand \& Wortmann, 1981, p 228).

This simple FFL/BFL procedure follows previous studies in the sense that the entire processing time of the operation must be loaded into the time bucket (e.g. Bobrowski, 1989; Cigolini et al., 1998). This avoids that the workload that is already loaded in a time bucket is pushed forward, if no pre-emption is allowed. However, at the same time, this arguably represents a first major weakness (Thürer et al., 2013). A second major weakness is that the workload distribution is based on the scheduled workload only - feedback regarding deviations from the schedule that occur on the shop floor is neglected. As a consequence, two additional forward finite loading rules that address these two weaknesses can be identified in

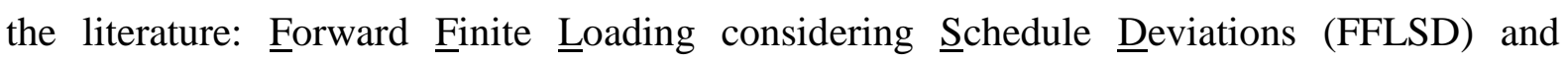

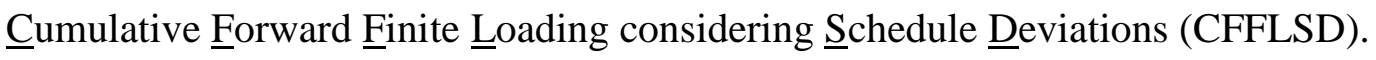

- FFLSD applies the same methodology as FFL except that the schedule deviation or 'backlog' is considered. This methodology is similar to the one applied by Kim \& 
Bobrowski (1995) in the context of order release to account for the positive backlog. In our study, both positive and negative backlogs are considered where a backlog is defined as the load that should have been completed in the past minus the load that has actually been completed. The backlog is added to the currently loaded work for calculation by distributing it over the time buckets.

- CFFLSD is based on the work of Bertrand (1983a, 1983b). It is equivalent to FFLSD, but a cumulative load is applied. This allows the load of an operation to be spread across multiple time buckets. The load of each operation contributes to the cumulative load until it is complete. This accounts for schedule deviations: operations behind schedule contribute to all cumulative loads, while operations that are ahead of schedule are subtracted from the cumulative loads.

Finally, note that using the principles of FFLSD and CFFLSD in combination with backward finite loading (i.e. BFLSD and CBFLSD) is not meaningful for two main reasons. First, there is no consistent rule for determining how to distribute the backlog if FFLSD is applied backwards (Nyhuis \& Pereira Filho, 2002). Second, for CFFLSD, the distance between the cumulative workload and the capacity curve increases with time, since only a part of the future workload is confirmed; hence all operations are likely to be loaded close to the due date if this method is transformed into backward loading. As a result, while three forward finite loading alternatives have been identified - FFL, FFLSD, and CFFLSD - only one backward loading method will be considered in this study - BFL.

\subsubsection{If, When, and Where Should Capacity be Adjusted?}

There are arguably two ways of using finite loading to determine if capacity needs to be adjusted (Nyhuis \& Pereira Filho, 2002):

- Forward Scheduling, whereby a due date is calculated by forward scheduling from the current date. If this due date violates the due date given by the customer then capacity needs to be adjusted.

- Backward Scheduling, whereby a planned release date is calculated by backward scheduling from the due date given by the customer. If this planned release date lies in the past then capacity needs to be adjusted.

Meanwhile, the decision concerning when capacity should be adjusted is driven by two alternatives: (i) adjusting capacity as soon as possible; or, (ii) adjusting capacity just-in-time. Finally, in answer to the question concerning where capacity should be adjusted, capacity 
should arguably be adjusted at the station with the longest operation throughput time calculated by the forward-backward loading procedure, since this station is most likely to be the bottleneck (e.g. Bechte, 1994).

\subsection{Research Motivation}

Our literature review identified four different finite loading methods that can be used to guide short-term capacity adjustments in make-to-order shops. These methods are summarized in Table 1.

\section{[Take in Table 1]}

The performance impact of the different methods however remains unclear. While the literature has advocated finite loading as potential means of guiding capacity adjustments, their actual operational impact is yet to be adequately assessed. Rather, the use of finite loading has typically been restricted to determining due dates, guiding order acceptance decisions, identifying which jobs to subcontract, or guiding the order release decision. In response, this study asks:

RQ1: What is the best forward/backward finite loading method to guide capacity adjustments in the context of make-to-order production?

In addition to the above, a workload trigger method to guide capacity adjustments in make-to-order shops has recently been presented by Land et al. (2015). This method monitors the workload (both on its way and not yet completed at each station) and uses a triggering workload threshold to determine when and where to adjust capacity. This represents an alternative to finite loading. However, while this method was shown to be a simple and effective solution for capacity control in make-to-order job shops, it completely neglects the urgency of orders. Thus, a very costly capacity increase could potentially be triggered, although the due date of orders lies long enough into the future to allow for the higher workload to be accommodated without adjustment. In contrast, finite loading considers both capacity and the urgency of orders. Our second research question therefore asks:

RQ2: How does the performance of the best forward/backward finite loading method compare with that of a load trigger method (namely, Land et al., 2015)?

To answer these two questions, we will use a controlled simulation environment of a make-to-order job shop. 


\section{Simulation Model}

In Section 3.1, we outline how we model the different capacity control mechanisms before the dispatching rule that controls the progress of jobs on the shop floor is introduced in Section 3.2. The shop and job characteristics modeled in the simulations are then described in Section 3.3. Finally, the experimental setting is outlined and the measures used to evaluate performance are presented in Section 3.4.

\subsection{Capacity Control}

In this study, we are not interested in the specific adjustment mechanisms used (e.g. overtime or additional shifts) but in determining if, when, and where capacity should be adjusted. To model a capacity adjustment, we therefore follow Land et al. (2015) and simply decrease the operation processing time by a predetermined percentage $\alpha$. To assess the impact of this factor, four different scenarios for the adjustment size $\alpha$ are considered: 0 (i.e. no capacity adjustment), 10, 20, and a $30 \%$ reduction in the operation processing time.

\subsubsection{Finite Loading Mechanism}

To answer our first research question - What is the best forward/backward finite loading method to guide capacity adjustments in the context of make-to-order production? - all four finite loading methods identified from the literature review (see Table 1) are considered. As in previous simulation studies on finite loading (e.g. Bobrowski, 1989; Cigolini et al., 1998; Thürer et al., 2013) it is assumed that all materials are available, and all necessary information regarding shop floor routings, processing times, etc. is known. For forward finite loading, the following procedure is executed when the order arrives at the shop:

- A customer due date is calculated by forward finite loading.

- If the calculated due date exceeds the given (customer) due date then capacity adjustments are required. Otherwise, the order's processing times are loaded into the corresponding time buckets and the procedure is complete.

- If capacity needs to be adjusted, the where and when questions need to be answered. The capacity adjustment should take place at the station which is most likely to be the bottleneck. As an indicator, we use the operation throughput time calculated by the forward finite loading procedure (Bechte, 1994). In other words, capacity is adjusted at the station with the longest estimated operation throughput time. Similar to Land et al. (2015), capacity adjustments are started as early as possible; this is with the next imminent time 
bucket. If a capacity adjustment is already active at the station in this time bucket, the adjustment duration is increased by considering the next time bucket until a time bucket without adjustment that lies before the calculated operation due date at this station is found. Hence, the maximum duration of adjustment is from the imminent time to the operation due date of the job. If such a time bucket is found, a new due date is calculated considering the additional capacity adjustments and the above steps are repeated. Otherwise, the procedure is complete and the job could not be fit into the station's capacity. Once the procedure is complete, the job is released onto the shop floor.

If capacity is adjusted in a time bucket then the workload loaded in the time bucket is reduced by the adjustment size $\alpha$. Increasing the duration of the adjustment stepwise increases the likelihood of the job's workload being fit. At the end of the procedure, there are three options: the job did not require any capacity adjustments (and therefore did not need to be fit), the job required capacity adjustments and could be fit, and the job required capacity adjustments but could not be fit. If there would be no time period (of the size of the lead time allowance) in which the incoming workload exceeds the capacity (i.e. the job cannot be fit) then the percentage tardy and mean tardiness would be zero. Yet Land et al. (2015) showed that, in the kind of job shop environment simulated in our study, significant overload situations necessarily occur. Performance differences across control solutions are determined by the timeliness and strength of response to these overload situations.

For backward finite loading, the same procedure is applied but backwards; hence capacity is adjusted if the planned release date is in the past. The time bucket for FFL, FFLSD, and BFL is set to 4 time units, which is the maximum processing time. The capacity norm for FFL is set to 4.1 and for FFLSD and BFL to 4.5 time units. These parameters are based on preliminary simulation experiments and result in the best overall performance. The capacity norm is larger than the time bucket size to allow for the granularity of the loaded workload. For CFFLSD, the time bucket size is arbitrarily set to 1 time unit. Additional simulation experiments showed no significant differences compared to the use of smaller time buckets. Meanwhile, four settings are considered for the minimum time allowance for the operation throughput time: $0,1,2$, and 3 time units.

\subsubsection{Reference Methods}

To answer our second research question - How does the performance of the best forward/backward finite loading method compare with that of a load trigger method (namely, Land et al., 2015)? - we introduce the procedure outlined by Land et al. (2015) as a 
reference method. The if, when, and where dimensions of the capacity adjustment are determined based on two load thresholds: (i) a load threshold that triggers the commencement of the capacity adjustment ( $\beta$ ); and, (ii) a load threshold that signals the load has reduced sufficiently to cease the adjustment $(\gamma)$. In this study, we only use one level of $\beta$ and one level of $\gamma$, which is justified by the performance frontier observed in Land et al. (2015). The threshold values have been determined numerically based on preliminary simulation experiments. For each station, we recorded the cumulative frequency distribution of the workload that is still to be completed at a station (measured in terms of the corrected load) that emerges without capacity adjustment. The corrected load divides the workload contribution of a job at a particular station by the position of the station in the routing of a job. It gives the best representation of the future expected direct load of a station based on the mix of routings actually present on the shop floor (Oosterman et al., 2000). The load threshold was then derived using a percentile of this distribution: for $\beta$ the $90^{\text {th }}$ percentile $(\beta=18.32)$ and for $\gamma$ the $85^{\text {th }}$ percentile $(\gamma=16.02)$.

Finally, and as a control, we also consider a general increase in capacity at each station, i.e. without specific consideration for if, when, and where capacity should be adjusted.

\subsection{Priority Dispatching}

This study focuses on the performance of the finite loading methods; therefore, once the finite loading procedure is executed, the order enters the shop floor. In other words, jobs are released immediately, and no specific order release procedure is executed. For capacity control to be effective, the dispatching rule applied on the shop floor should use the operation due dates or operation start dates determined by the capacity control mechanism. This ensures that capacity control takes place, i.e. that capacity is used as planned.

Since the reference capacity control method from Land et al. (2015) does not calculate operation due dates, the operation due dates are calculated by successively backward scheduling a constant allowance for the operation throughput time of each operation in the routing of a job. This constant allowance was set to 3 time units, since this setting resulted in the best overall performance in preliminary simulation experiments. The same procedure was used for our second reference method considering a general increase in capacity.

\subsection{Overview of Modeled Shop and Job Characteristics}

A simple job shop model is used to avoid interactions that may interfere with our understanding of the effects of the experimental factors. A simulation model of a randomly routed job shop (Conway et al., 1967) - later referred to as a pure job shop (Melnyk \& 
Ragatz, 1989) - has been implemented in the Python ${ }^{\odot}$ programming language using the $\mathrm{SimPy}^{\odot}$ simulation module. The model is stochastic, whereby job routings, processing times, inter-arrival times and due dates are stochastic (random) variables. We did not consider rush orders given that the impact of rush orders has been assessed previously in Thürer et al. (2010) and, more recently, in Jäger \& Roser (2018). A generally accepted rule of thumb is that the amount of prioritized workload should be below 30\%. We also did not consider machine breakdown or quality issues since creating additional uncertainty via differences between the estimated and realized processing time did not significantly influence the relative performance of the forward finite loading methods in Thürer et al. (2013).

The shop contains six stations, where each station is a single, constant capacity resource. The routing length of jobs varies uniformly from one to six operations. All stations have an equal probability of being visited and a particular station is required at most once in the routing of a job. Operation processing times follow a truncated 2-Erlang distribution with a mean of 1 time unit after truncation and a maximum of 4 time units. The inter-arrival time of jobs follows an exponential distribution with a mean of 0.648 , which - based on the average number of stations in the routing of a job - deliberately results in a utilization level of $90 \%$ without capacity adjustments. Due dates are set exogenously by adding a uniformly distributed random allowance factor. Three levels of due date tightness are considered: tight due dates, with an allowance factor between 28 and 36 time units; medium due dates, with an allowance factor between 32 and 40 time units; and loose due dates, with an allowance factor between 36 and 44 time units.

The modelled shop and job characteristics are summarized in Table 2. While any individual job shop in practice will differ in many aspects from this stylized environment, it captures the typical job shop characteristics of high routing variability, processing time variability, and arrival variability.

[Take in Table 2]

\subsection{Experimental Design and Performance Measures}

The (main) experimental factors are summarized in Table 3. A full factorial design was used with $192(4 \times 4 \times 4 \times 3)$ scenarios. In addition to the full factorial design, we also included experiments with the load trigger method presented in Land et al. (2015) and experiments with a general increase in capacity. Each scenario was replicated 100 times while results were collected over 10,000 time units following a warm-up period of 3,000 time units. These parameters are in line with those used in previous studies that have applied similar job shop 
models and allow us to obtain stable results while keeping the simulation run time reasonable.

\section{[Take in Table 3]}

Since we focus on a make-to-order shop, our main performance indicator will be delivery performance. This is motivated by the fact that although the underlying concern of a firm may be cost, it is important to recognize that cost structures are firm-specific; hence, it is extremely difficult to objectively evaluate the performance of the different rules based on cost. The best rule to choose will depend on a firm's idiosyncratic cost structure. Delivery performance will be measured by: the percentage tardy - the percentage of jobs completed after the due date; and, the mean tardiness, that is $T_{j}=\max \left(0, L_{j}\right)$, with $L_{j}$ being the lateness of job $j$ (i.e. the actual delivery date minus the due date of job $j$ ). In addition, we also measure the mean lead time, i.e. the mean of the completion date minus the entry date across jobs. The mean lead time also reflects the average workload in the system. In order to measure the capacity increase that is incurred in order to realize a given level of delivery performance, we measure the average utilization level.

\section{Results}

An Analysis of Variance (ANOVA) has been used to obtain a first indication of the relative impact of the experimental factors. The ANOVA is here based on a block design, which is typically used to account for known sources of variation in an experiment. In our ANOVA, we treat due date tightness as the blocking factor. This allows the main effects of this environmental factor and the main and interaction effects of our other three finite loading related factors (finite loading method, minimum allowance, and adjustment size) to be captured. The results are presented in Table 4.

\section{[Take in Table 4]}

All main effects, two-way interactions, and the three-way interaction were shown to be statistically significant. To obtain a first indication of the direction and size of the performance differences between the different finite loading methods, the Scheffé multiple comparison procedure was applied. The results in Table 5 suggest that CFFLSD has the potential to outperform the three other finite loading methods. To further assess performance differences, detailed performance results will be presented next in Section 4.1 where we 
assess performance under different levels of due date tightness. A performance analysis is then provided in Section 4.2. Finally, the impact of the minimum allowance is assessed in Section 4.3.

[Take in Table 5]

\subsection{Performance Assessment}

A major challenge when comparing different control methods is the creation of comparable states - a certain parameter setting may favor one method over another thereby making conclusions dependent on parameter settings rather than on the actual performance of the different methods applied. A means of realizing a 'fair' comparison is via the use of operating characteristic curves (Olhager \& Persson, 2008). Rather than comparing one specific parameter setting, parameters are varied for each control method and the results are presented in the form of performance curves. The relative positioning of the different curves (each representing one control method) then allows the relative performance of each method to be compared. In our study, the left-hand starting point of the curves represents the highest level of alpha. The level of alpha decreases step-wise by moving from left to right in each graph, with each data point representing one alpha level (i.e. a 30\%, 20\%, 10\%, and $0 \%$ capacity adjustment). Decreasing alpha reduces the total overall amount of the capacity adjustment and, as a result, increases the utilization level. This utilization level is an indicator of the capacity adjustment required to achieve a certain performance improvement.

Figure 1 shows the percentage tardy, mean tardiness, and lead time over the resulting average utilization level. Figure 1a gives the results for a tight due date, Figure $1 \mathrm{~b}$ for a medium due date, and Figure 1c for a loose due date. Only results for a minimum allowance of zero are shown, with the impact of this allowance being assessed in the next section.

\section{[Take in Figure 1]}

In addition to the four curves in Figure 1 representing the four different finite loading methods, we provide one further graph that gives the results obtained using the load trigger method presented in Land et al. (2015) and one graph that gives the results for a general increase in capacity, i.e. without specific consideration for when and where capacity should be adjusted, referred to as the "all adjusted" method. The following can be observed from the results:

- General Performance of Finite Loading Methods: If the curve of a finite loading method lies below the dashed line then performance is better than when a general increase in 
capacity is applied (i.e. our baseline comparison). We can observe that all finite loading methods have the potential to improve performance in terms of the percentage tardy and mean tardiness if the adjustment size is set appropriately. CFFLSD leads to the best performance. FFL and BFL realize a similar level of mean tardiness, although neither considers feedback from the shop floor. In terms of the lead time, BFL performs the worst due to the backward scheduling of operation due dates. This means that BFL improves tardiness performance through a reduction in the standard deviation of lateness.

- Finite Loading Methods vs. The Load Trigger Method: Our results demonstrate that a far superior level of performance can be achieved in terms of percentage tardy and mean tardiness by the load trigger method from Land et al. (2015). Similar to Wein \& Chevallier (1992) in the context of job shop scheduling, better due date performance can be achieved by ignoring due dates on the shop floor.

- The Impact of Due Date Tightness: The impact of due date tightness can be observed by moving from Figure 1a (tight due dates) to Figure 1c (loose due dates). While there is (as expected) a general improvement in tardiness performance, the relative performance impact of the different finite loading methods is not affected. We can also observe a shift to the right, which indicates that fewer jobs require capacity adjustments if due dates increase, i.e. delivery lead times become longer.

\subsection{Performance Analysis}

This section seeks to gain a better understanding into where each method has its strongholds and where it performs weakly, taking into account three criteria: the capability to identify a tardy order correctly, the capability to adjust capacity so an order can be fit into the schedule, and the effectiveness of these adjustments, i.e. how many of the fitted orders are actually delivered on time. To evaluate each criterion, we collected data on the following four measures: (i) the percentage of orders that needed a capacity adjustment according to the finite loading method (referred to as "need adj."); (ii) the percentage of tardy orders that were correctly identified by the finite loading method (referred to as "tardy id."); (iii) the percentage of orders that needed to be fit and could eventually be fit (referred to as "fit"); and, (iv) the percentage of orders which could be fit and that could be delivered on time ("on time"). All four measures are given in Table 6.

[Take in Table 6] 
If we focus on the scenario where alpha is zero, i.e. no capacity adjustments are made, then we can observe that FFL and CFFLSD obtain the most accurate predictions that an order will be tardy, with around $80 \%$ of the tardy orders being correctly identified. If we look at the percentage of orders that could actually be fit, then we observe substantial differences between FFL and CFFLSD, whereby CFFLSD is able to fit significantly more orders within the available capacity. However, this does not mean that all of these orders are delivered on time. While, under FFL, almost $100 \%$ of orders that are fit are also delivered on time, this is not the case for CFFLSD. In fact, CFFLSD is the worst performing finite loading method in terms of this measure ("on time"), although the total amount of orders delivered tardy is still below the other methods. One possible explanation is the cumulative measure that allows for a tighter, less granular schedule; in other words, no buffers are introduced. In contrast, the granular schedule of FFL introduces buffers. The importance of granularity and the associated buffer is also highlighted by the results for BFL. While BFL is by far the worst performing method in terms of predicting whether an order will be tardy ("tardy id."), more than $90 \%$ of the orders that were fit could be delivered on time.

\subsection{The Impact of the Minimum Allowance}

Figure 2 shows the percentage tardy, mean tardiness, and lead time over the resulting average utilization level for the different levels of the minimum allowance. Figure 2a gives the results for an allowance of 1 time unit, Figure $2 b$ for an allowance of 2 time units, and Figure $2 c$ for an allowance of 3 time units. Only results for a medium due date tightness are shown here as the performance impact is similar across the three different levels of this factor.

\section{[Take in Figure 2]}

As somewhat expected, if the minimum allowance is increased (i.e. by moving from Figure 2a to Figure 2c) stronger capacity adjustments are realized, i.e. the graphs extend to the left. The following can be observed from the results:

- General Performance of Finite Loading Methods: FFL and especially FFLSD improve in performance as the minimum allowance is increased. For example, FFLSD improves performance from $10 \%$ tardy to $5 \%$ tardy at a utilization level of $89 \%$.

- Finite Loading Methods vs. The Load Trigger Method: Our results demonstrate that the far superior level of performance that can be achieved in terms of percentage tardy and mean tardiness by the load trigger method from Land et al. (2015) is maintained. 
To better understand the improvement for FFL and FFLSD, we monitored the development of the corrected workload and the adjustment periods over time. We focused on the corrected workload since this is the best estimate of the future direct load when a job enters the shop floor (Oosterman et al., 2000). Figure 3 gives the results for FFL and an arbitrary chosen work center based on 7,000 time units of a representative simulation run. Focusing on the overload period around 5,000 time units, we observe that the connectedness of the adjustment period that occurs at a minimum allowance of 2 significantly reduces the impact of this overload period. Increasing the minimum allowance increases the likelihood that an order is loaded into the next time bucket and consequently the estimated due dates. This in turn increases the adjustment periods.

\section{[Take in Figure 3]}

FFLSD shows similar behavior for the overload period, but the method shows less overreaction in periods with lower loads since it considers the backlog. Thus, it realizes the performance improvement with a lesser amount of capacity adjustment. Compared to FFL, the number of adjustments reduces from XXX to XXX and the duration reduces from $\mathrm{XXX}$ to XXX. The best performance is achieved by the load trigger method, which shows no overreaction but only adjusts capacity during overload periods. This can be observed from Figure 4, which gives the overtime results for the remaining capacity adjustment methods: FFLSD, CFFLSD, BFL and the load trigger method. For FFLSD, CFFLSD, and BFL the results for a minimum allowance of 3 are given. Meanwhile, CFFLSD is not able to reduce the overload period. An explanation for this is that there are periods in which no capacity adjustments take place since CFFLSD realizes a better fit.

\section{[Take in Figure 4]}

The main difference between the load trigger method and the finite loading methods is that, for the latter, it is the individual jobs that trigger the adjustments whereas, for the former, the aggregate of the workload of all jobs on the shop floor triggers the adjustment. If the individual job triggers the adjustments, then one of the following may occur:

- Capacity adjustments are not triggered although there is an overload period (this can be observed by adjustments that are not sustained). For example, jobs with one operation and a large due date, or large jobs with only one overloaded station in their routing, are fit into their due dates although there is an overload period. This results in a performance loss compared to the load trigger method. 
- Capacity adjustments are triggered although there is no overload period since, e.g. a large job with a tight due date cannot be fit. This results in more capacity adjustments compared to the load trigger method.

Increasing the minimum allowance or the due date tightness reduces the risk of not making necessary adjustments during overload periods but increases the risk of unnecessary adjustments when there is no overload. If finite loading methods sustain the adjustment over the whole overload period then performance is similar to the load trigger method. But this parity of performance only happens at the cost of many unnecessary capacity adjustments; hence, the total amount of realized capacity adjustment is increased.

\section{Conclusions}

Capacity management is an important production control function that significantly influences firm performance. It spans from long-term capacity planning through to short-term capacity control. However, most capacity management research to date has focused on longterm or medium-term capacity decisions in the context of known or deterministic customer demand. There has been only limited attention on short-term capacity management, especially in a make-to-order context where demand is uncertain or non-deterministic. In such a context, the optimization algorithms that dominate the literature are arguably not feasible given, for example, that the optimization procedure would have to be re-executed every time a new job arrived at the shop.

One method specifically designed to accomplish the challenging task of guiding shortterm capacity adjustments in a high-variety, make-to-order context is the use of finite loading. However, while research has developed this method theoretically, the actual performance impact of finite loading methods has not been adequately assessed. In response, our first research question asked: What is the best forward/backward finite loading method to guide capacity adjustments in the context of make-to-order production? Our simulation results have demonstrated that a forward finite loading mechanism that considers schedule deviations, i.e. FFLSD, has the potential to outperform all other methods if the minimum allowance is set appropriately. Yet, more fundamentally, our simulation results draw into question the use of finite loading as advocated in the literature. In answer to our second research question - How does the performance of the best forward/backward finite loading method compare with that of a load trigger method (namely, Land et al., 2015)? - our simulation results have demonstrated that all four methods are outperformed by the load 
trigger method from Land et al. (2015). Both findings have important implications for practice and future research.

\subsection{Managerial Implications}

Forward finite loading that considers schedule deviations outperformed all other finite loading methods in our study. But already the simplest form of forward and backward finite loading methods, which do not consider any feedback from the shop floor, has the potential to improve performance compared to a general capacity increase. This highlights the potential of transferring from infinite loading via the use of lead time off-sets, as typically implemented in MRP/ERP systems, to finite loading. However, at the same time, our study has questioned the use of finite loading in its current form as a load trigger method outperformed all finite loading methods identified from the literature. These results suggest that this method should be the first choice in high-variety make-to-order shops in practice. Rather than scheduling operation throughput times under capacity constraints the workload is directly measured and monitored, using this workload information to inform and guide production control decisions. In other words, our results reemphasize Bertrand \& Wortmann (1981) in that the proper modeling and measuring of the workload should be the bedrock of production control.

\subsection{Limitations and Future Research}

This study has focused on finite loading methods. Hence, a limitation is its neglect of other means of considering urgency in capacity adjustment decisions. Further, our focus has been on make-to-order job shops characterized by three stochastic elements: the inter-arrival time, the routing, and processing times. Finite loading methods are likely to be more effective in repetitive environments. More repetitive environments would also allow for the application of optimization based approaches, which were neglected in our study due to its highly stochastic context. Hence, future research could compare the performance of finite loading methods with optimization based approaches in more repetitive production environments, considering potential trade-offs between performance and solution complexity. Finally, we observed that one of the main weaknesses of finite loading is that it does not sustain the capacity adjustment during an overload period. The adjustment can only be sustained if an overreaction during underload periods is accepted. The main cause is that, for finite loading, the capacity adjustment depends on individual jobs and their properties, such as the due date or routing. Hence, an adjustment may not be triggered in an overload period since there is only one overloaded station in the routing of a job or an adjustment may be triggered 
unnecessarily since a large job has a tight due date. The former deteriorates performance and the latter increases the amount of capacity adjustments compared to the load trigger method. Future research could build on this finding and develop new methods that consider different measures for integrating urgency considerations into the capacity adjustment decision, e.g. based on expected lateness (Van Ooijen \& Bertrand, 2018).

\section{References}

Ahmed, I., \& Fisher, W.W., 1992, Due date assignment, job order release and sequencing interaction in job shop scheduling, Decision Sciences, 23, 633 - 647.

Bechte, W., 1994, Load-oriented manufacturing control just-in-time production for job shops, Production Planning and Control, 5, 3, 292 - 307.

Bertrand, J.W.M, 1983a, The use of workload information to control job lateness in controlled and uncontrolled release production systems, Journal of Operations Management, 3, 2, $79-92$.

Bertrand, J.W.M, 1983b, The effect of workload dependent due-dates on job-shop performance, Management Science, 29, 7, 799 - 816.

Bertrand, J.W.M., and Wortmann, J.C., 1981, Production control and information systems for component-manufacturing shops, Elsevier Scientific Publishing Company, Amsterdam.

Bobrowski, P.M., 1989, Implementing a loading heuristic in a discrete release job shop, International Journal of Production Research, 27, 11, 1935-1948.

Cigolini, R., Perona, M., \& Portioli, A., 1998, Comparison of Order Review and Release techniques in a dynamic and uncertain job shop environment, International Journal of Production Research, 36, 11, 2931 - 2951.

Chen, K., \& Ji, P., 2007, A mixed integer programming model for advanced planning and scheduling (APS), European Journal of Operational Research, 181, 515-522.

Chen, Z.L., Li, S., \& Tirupati, D., 2002, A scenario-based stochastic programming approach for technology and capacity planning, Computers \& Operations Research, 29, 781-806.

Cigolini, R., Perona, M., \& Portioli, A., 1998, Comparison of Order Review and Release techniques in a dynamic and uncertain job shop environment, International Journal of Production Research, 36, 11, 2931 - 2951.

Conway, R., Maxwell, W.L., \& Miller, L.W., 1967, Theory of Scheduling, Reading, MA: Addisson-Wesley. 
Corti, D., Pozzetti, A., \& Zorzini, M., 2006, A capacity-driven approach to establish reliable due dates in a MTO environment, International Journal of Production Economics, 104, $536-554$.

Geng, N., \& Jiang, Z., 2009, A review on strategic capacity planning for the semiconductor manufacturing industry, International Journal of Production Research, 47, 13, 36393655.

Geng, N., Jiang, Z., \& Chen, F., 2009, Stochastic programming based capacity planning for semiconductor wafer fab with uncertain demand and capacity, European Journal of Operational Research, 198, 899-908.

Hendry, L.C., Kingsman, B.G., \& Cheung, P., 1998, The effect of workload control (WLC) on performance in make-to-order companies, Journal of Operations Management, 16, 63 75 .

Hendry, L.C., \& Kingsman, B.G., 1993, Customer Enquiry Management: Part of a hierarchical system to control lead times in make-to-order companies, Journal of the Operational Research Society, 44, 1, $61-70$.

Jäger, Y., and Roser, C., Effect of Prioritization on the Waiting Time, APMS Conference 2018.

Kim, S.J., \& Bobrowski, P.M., 1995, Evaluating order release mechanisms in a job shop with sequence-dependent set-up times, Production and Operations Management, 4, 2, 163 180.

Kingsman, B.G., \& Hendry, L.C., 2002, The relative contributions of input and output controls on the performance of a workload control system in Make-To-Order companies, Production Planning and Control, 13, 7, 579-590.

Kingsman, B.G., Tatsiopoulos, I.P., \& Hendry, L.C., 1989, A structural methodology for managing manufacturing lead times in make-to-order companies, European Journal of Operational Research, 40, 196 - 209.

Land, M.J., Stevenson, M., Thürer, M., \& Gaalman, G.J.C., 2015; Job Shop Control: In Search of the Key to Delivery Improvements, International Journal of Production Economics, 168, 257-266.

Lin, J.T. Chen, T.L., \& Chu, H.C., 2014, A stochastic dynamic programming approach for multi-site capacity planning in TFT-LCD manufacturing under demand uncertainty, International Journal of Production Economics, 148, 21-36.

Lödding, H., 2012, A manufacturing control model, International Journal of Production Research, 50, 22, 63311-6328. 
Martínez-Costa, C., Mas-Machuca, M., Benedito, E., \& Corominas, A., A review of mathematical programming models for strategic capacity planning in manufacturing, International Journal of Production Economics, 153, 66-85.

Melnyk, S.A., \& Ragatz, G.L., 1989, Order review/release: research issues and perspectives, International Journal of Production Research, 27, 7, 1081-1096.

Moses, S., Grant, H., Gruenwald, L., \& Pulat, S., 2004, Real-time due-date promising by build-to-order environments, International Journal of Production Research, 42-20, 4353 4375 .

Nyhuis, F., \& Pereira Filho, 2002, Methods and tools for dynamic capacity planning and control, Gestão \& Produção, 9, 33, 245-260.

Olhager J., \& Persson F., 2008, Using Simulation-Generated Operating Characteristics Curves for Manufacturing Improvement, In: Koch T. (eds) Lean Business Systems and Beyond. IFIP - The International Federation for Information Processing, 257, Springer, Boston, MA

Olhager, J., Rudberg, M., \& Wikner, J., 2001, Long-term capacity management: Linking the perspectives from manufacturing strategy and sales and operations planning, International Journal of Production Economics, 69, 215-225.

Oosterman, B., Land, M.J., \& Gaalman, G., 2000, The influence of shop characteristics on workload control, International Journal of Production Economics, 68, 1, 107-119.

Ragatz, G. L., \& Mabert, V. A., 1988, An evaluation of order release mechanisms in a job shop environment, Decision Sciences, 19, 167 - 189.

Rossi, T., Pozzi, R., Pero, M., \& Cigolini, R., 2016, Improving production planning through finite-capacity MRP, International Journal of Production Research, 55, 2, 377-391.

Tenhiälä, A., Contingency theory of capacity planning: The link between process types and planning methods, Journal of Operations Management, 29, 65-77.

Thürer, M., Silva, C., and Stevenson, M., 2010, Workload Control Release Mechanisms: From Practice back to Theory Building, International Journal of Production Research, 48, $12,3593-3617$.

Thürer, M., Stevenson, M., Qu, T., \& Godinho Filho, M., 2014, The Design of Simple Subcontracting Rules for Make-to-Order Shops: An Assessment by Simulation, European Journal of Operational Research, 239, 854-864.

Thürer, M., Stevenson, M., Silva, C., \& Land, M.J., 2013, Towards an Integrated Workload Control (WLC) Concept: The Performance of Due Date Setting Rules in Job Shops with Contingent Orders, International Journal of Production Research, 51, 15, 4502-4516. 
Van Ooijen, H.P.G., \& Bertrand, J.W.M., 2018, The Effect of Due-Date driven Capacity Adjustments on Delivery Performance and Costs in Job Shops with Exogenous Due-Dates, $20^{\text {th }}$ International Working Seminar on Production Economics, Innsbruck, Austria, 347353

Wiendahl, H.P., 1995, Load oriented manufacturing control, Springer Verlag, Berlin Heidelberg, New York.

Wortmann, J.C., Euwe, M.J., Taal, M. \& Wiers, V.C.S. ,1996, A review of capacity planning techniques within standard software packages, Production Planning and Control, 7, 2, 117-128. 
Table 1: Summary of Forward and Backward Finite Loading Procedures from the Literature

\begin{tabular}{|c|c|c|c|}
\hline Category & Acronym & Name of Rule & Brief Description \\
\hline \multirow{3}{*}{$\begin{array}{l}\text { Forward } \\
\text { Finite } \\
\text { Loading }\end{array}$} & FFL & $\begin{array}{l}\text { Forward Finite } \\
\text { Loading }\end{array}$ & $\begin{array}{l}\text { Operation due dates are determined step by step, fitting } \\
\text { operations to the remaining capacity. The planning } \\
\text { horizon is broken down into time buckets. An operation } \\
\text { is scheduled into the first time bucket with sufficient } \\
\text { capacity after the previous operation due date and } \\
\text { considering a minimum flow time allowance. The } \\
\text { operation due date of the last operation determines the } \\
\text { job due date. }\end{array}$ \\
\hline & FFLSD & $\begin{array}{l}\text { FFL considering } \\
\text { Schedule } \\
\text { Deviations }\end{array}$ & $\begin{array}{l}\text { As for FFL but considering schedule deviations. The } \\
\text { backlog resulting from schedule deviations is distributed } \\
\text { over the time buckets. }\end{array}$ \\
\hline & CFFLSD & $\begin{array}{l}\text { Cumulative FFL } \\
\text { considering } \\
\text { Schedule } \\
\text { Deviations }\end{array}$ & $\begin{array}{l}\text { As for FFLSD but operation due dates are determined by } \\
\text { fitting the cumulative workload to the cumulative } \\
\text { capacity. }\end{array}$ \\
\hline $\begin{array}{l}\text { Backward } \\
\text { Finite } \\
\text { Loading }\end{array}$ & BFFL & $\begin{array}{l}\text { Backward Finite } \\
\text { Loading }\end{array}$ & $\begin{array}{l}\text { As for FFL but backwards. Operation start dates are } \\
\text { determined step by step, fitting operations to the } \\
\text { remaining capacity. The planning horizon is broken } \\
\text { down into time buckets. An operation is scheduled into } \\
\text { the first available time bucket with sufficient capacity. } \\
\text { The start date of the first operation determines the } \\
\text { planned release date. }\end{array}$ \\
\hline
\end{tabular}

Table 2: Summary of Simulated Shop and Job Characteristics

\begin{tabular}{|c|c|c|}
\hline & $\begin{array}{r}\text { Routing Variability } \\
\text { No. of Stations } \\
\text { Interchange-ability of Stations } \\
\text { Station Capacities }\end{array}$ & $\begin{array}{l}\text { Random routing; no-re-entrant flows } \\
6 \\
\text { No interchange-ability } \\
\text { All equal; output control is exercised }\end{array}$ \\
\hline 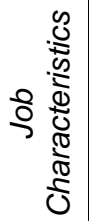 & $\begin{array}{r}\text { No. of Operations per Job } \\
\text { Operation Processing Times } \\
\text { Due Date Determination Procedure } \\
\text { Inter-Arrival Times }\end{array}$ & $\begin{array}{l}\text { Discrete Uniform[1, 6] } \\
\text { Truncated 2-Erlang; }(\operatorname{mean}=1 ; \max =4) \\
\text { Due Date = Entry Time+ } d ; d U \sim[28,36],[32,40],[36,44] \\
\text { Exp. Distribution; mean }=0.648\end{array}$ \\
\hline
\end{tabular}

Table 3: Experimental Settings

\begin{tabular}{r|l}
\hline Factors & Levels \\
\hline Finite Loading Method & FFL, FFLSD, CFFLSD, and BFL \\
Minimum Allowance & $0,1,2$ and 3 time units \\
Adjustment Size ( $\alpha)$ & $0,10,20$ and $30 \%$ \\
Due Date Tightness [time units] & Tight $[28,36]$, Medium $[32,40]$ and Loose due dates $[36,44]$ \\
\hline
\end{tabular}


Table 4: ANOVA Results

\begin{tabular}{|c|c|c|c|c|c|c|}
\hline & Source of Variance & $\begin{array}{r}\text { Sum of } \\
\text { Squares }\end{array}$ & $\begin{array}{l}\text { Degree of } \\
\text { Freedom }\end{array}$ & $\begin{array}{r}\text { Mean } \\
\text { Squares }\end{array}$ & F-Ratio & $\begin{array}{c}p- \\
\text { value }\end{array}$ \\
\hline \multirow{9}{*}{$\begin{array}{c}\text { Percentage } \\
\text { Tardy }\end{array}$} & Due Date Tightness & 12.52 & 2 & 6.26 & 5862.39 & 0.00 \\
\hline & Finite Loading (FL) & 10.00 & 3 & 3.33 & 3121.89 & 0.00 \\
\hline & Minimum Allowance (A) & 5.85 & 3 & 1.95 & 1825.17 & 0.00 \\
\hline & Adjustment Size (Alpha) & 42.04 & 3 & 14.01 & 13120.14 & 0.00 \\
\hline & $F L \times A$ & 1.50 & 9 & 0.17 & 156.27 & 0.00 \\
\hline & FL x Alpha & 2.99 & 9 & 0.33 & 311.46 & 0.00 \\
\hline & Allowance x Alpha & 1.95 & 9 & 0.22 & 202.59 & 0.00 \\
\hline & FL x Allowance $\times$ Alpha & 0.17 & 27 & 0.01 & 5.84 & 0.00 \\
\hline & Residual & 20.43 & 19134 & 0.00 & & \\
\hline \multirow{9}{*}{$\begin{array}{c}\text { Mean } \\
\text { Tardiness }\end{array}$} & Due Date Tightness & 961.83 & 2 & 480.92 & 1920.32 & 0.00 \\
\hline & Finite Loading (FL) & 535.60 & 3 & 178.53 & 712.89 & 0.00 \\
\hline & Minimum Allowance (A) & 301.87 & 3 & 100.62 & 401.79 & 0.00 \\
\hline & Adjustment Size (Alpha) & 6133.15 & 3 & 2044.38 & 8163.31 & 0.00 \\
\hline & $F L \times A$ & 128.22 & 9 & 14.25 & 56.89 & 0.00 \\
\hline & FL x Alpha & 564.57 & 9 & 62.73 & 250.48 & 0.00 \\
\hline & Allowance $\mathrm{x}$ Alpha & 127.21 & 9 & 14.13 & 56.44 & 0.00 \\
\hline & FL x Allowance $\times$ Alpha & 15.24 & 27 & 0.56 & 2.25 & 0.00 \\
\hline & Residual & 4791.83 & 19134 & 0.25 & & \\
\hline \multirow{9}{*}{ Lead Time } & Due Date Tightness & 6128.11 & 2 & 3064.05 & 1899.87 & 0.00 \\
\hline & Finite Loading (FL) & 62980.66 & 3 & 20993.55 & 13017.09 & 0.00 \\
\hline & Minimum Allowance (A) & 19040.15 & 3 & 6346.72 & 3935.29 & 0.00 \\
\hline & Adjustment Size (Alpha) & 68503.69 & 3 & 22834.56 & 14158.61 & 0.00 \\
\hline & $F L \times A$ & 4273.30 & 9 & 474.81 & 294.41 & 0.00 \\
\hline & FL x Alpha & 3781.14 & 9 & 420.13 & 260.50 & 0.00 \\
\hline & Allowance $x$ Alpha & 4880.10 & 9 & 542.23 & 336.21 & 0.00 \\
\hline & FL x Allowance $\times$ Alpha & 255.36 & 27 & 9.46 & 5.86 & 0.00 \\
\hline & Residual & 30858.71 & 19134 & 1.61 & & \\
\hline
\end{tabular}

Table 5: Results for the Scheffé Multiple Comparison Procedure

\begin{tabular}{|c|c|c|c|c|c|c|c|}
\hline \multirow{2}{*}{$\begin{array}{l}\text { FL } \\
\text { Method (x) }\end{array}$} & \multirow{2}{*}{$\begin{array}{l}\text { FL } \\
\text { Method (y) }\end{array}$} & \multicolumn{2}{|c|}{$\begin{array}{l}\text { Percentage } \\
\text { Tardy }\end{array}$} & \multicolumn{2}{|c|}{$\begin{array}{c}\text { Mean } \\
\text { Tardiness }\end{array}$} & \multicolumn{2}{|c|}{ Lead Time } \\
\hline & & lower1) & upper & lower & upper & lower & upper \\
\hline FFLSD & FFL & 0.035 & 0.039 & 0.232 & 0.289 & 2.414 & 2.559 \\
\hline CFLLSL & $\mathrm{FF}$ & -0.008 & -0.005 & $-0.014^{*}$ & 0.043 & -0.920 & -0.775 \\
\hline BFL & $\mathrm{FFL}$ & 0.044 & 0.048 & 0.366 & 0.423 & 3.554 & 3.699 \\
\hline CFFL & $\mathrm{FF}$ & -0.045 & -0.042 & -0.274 & -0.217 & -3.406 & -3.262 \\
\hline BFL & FFLSD & 0.008 & 0.011 & 0.106 & 0.163 & 1.068 & 1.213 \\
\hline BFL & CFFLSD & 0.051 & 0.055 & 0.352 & 0.409 & 4.402 & 4.547 \\
\hline
\end{tabular}

1) $95 \%$ confidence interval; ${ }^{*}$ not significant at $\alpha=0.05$ 
Table 6: Percentage of Orders that Needed to be Fit and the Orders that were Actually Fit for the Different Levels of Due Date Tightness

\begin{tabular}{|c|c|c|c|c|c|c|c|c|c|c|}
\hline \multirow[b]{2}{*}{ Method } & \multirow[b]{2}{*}{$\alpha$} & \multicolumn{3}{|c|}{ Tight Due Date } & \multicolumn{3}{|c|}{ Medium Due Date } & \multicolumn{3}{|c|}{ Loose Due Date } \\
\hline & & $\begin{array}{l}\text { need } \\
\text { adj. }\end{array}$ & $\begin{array}{c}\text { tardy } \\
\text { id. }\end{array}$ & $\begin{array}{l}\text { on } \\
\text { time }\end{array}$ & $\begin{array}{l}\text { need } \\
\text { adj. }\end{array}$ & $\begin{array}{c}\text { tardy } \\
\text { id. }\end{array}$ & $\begin{array}{l}\text { on } \\
\text { time }\end{array}$ & $\begin{array}{l}\text { need } \\
\text { adj. }\end{array}$ & $\begin{array}{c}\text { tardy } \\
\text { id. }\end{array}$ & $\begin{array}{l}\text { on } \\
\text { time }\end{array}$ \\
\hline FFL & 0 & $17.2 \%$ & $79.5 \%$ & $\mathrm{~N} / \mathrm{A}$ & $11.4 \%$ & $77.4 \%$ & $\mathrm{~N} / \mathrm{A}$ & $7.3 \%$ & $75.7 \%$ & $\mathrm{~N} / \mathrm{A}$ \\
\hline FFL & $10 \%$ & $8.4 \%$ & $69.2 \%$ & $2.0 \% \quad 95.4 \%$ & $5.4 \%$ & $67.2 \%$ & $2.0 \% \quad 95.8 \%$ & $3.5 \%$ & $65.4 \%$ & $1.9 \% \quad 96.9 \%$ \\
\hline FFL & $20 \%$ & $6.9 \%$ & $66.1 \%$ & $3.3 \% \quad 97.8 \%$ & $4.6 \%$ & $64.6 \%$ & $3.3 \% \quad 97.6 \%$ & $3.0 \%$ & $62.9 \%$ & $3.0 \% \quad 98.0 \%$ \\
\hline FFL & $30 \%$ & $6.4 \%$ & $65.0 \%$ & $4.0 \% \quad 98.7 \%$ & $4.4 \%$ & $64.2 \%$ & $3.7 \% \quad 98.5 \%$ & $2.9 \%$ & $61.6 \%$ & $3.6 \% \quad 98.6 \%$ \\
\hline FFL & 0 & $9.5 \%$ & $40.5 \%$ & $\mathrm{~N} / \mathrm{A}$ & $5.7 \%$ & $35.4 \%$ & $\mathrm{~N} / \mathrm{A}$ & $3.4 \%$ & $31.4 \%$ & $\mathrm{~N} / \mathrm{A}$ \\
\hline FFLSD & $10 \%$ & $5.0 \%$ & $28.9 \%$ & $2.1 \% \quad 48.0 \%$ & $3.2 \%$ & $25.8 \%$ & $2.0 \% \quad 47.4 \%$ & $1.9 \%$ & $23.2 \%$ & $2.0 \% \quad 51.1 \%$ \\
\hline FFLSD & $20 \%$ & $4.4 \%$ & $27.8 \%$ & $2.5 \% \quad 67.9 \%$ & $2.8 \%$ & $24.7 \%$ & $2.4 \% \quad 68.6 \%$ & $1.7 \%$ & $22.0 \%$ & $2.3 \% \quad 68.5 \%$ \\
\hline FFLSD & $30 \%$ & $4.2 \%$ & $27.1 \%$ & $2.6 \% \quad 77.8 \%$ & $2.6 \%$ & $24.2 \%$ & $2.4 \% \quad 80.7 \%$ & $1.7 \%$ & $21.8 \%$ & $2.1 \% \quad 80.1 \%$ \\
\hline CFFLSD & & $16.3 \%$ & $81.1 \%$ & $\mathrm{~N} / \mathrm{A}$ & $11.2 \%$ & $79.2 \%$ & $\mathrm{~N} / \mathrm{A}$ & $7.6 \%$ & $77.6 \%$ & $\mathrm{~N} / \mathrm{A}$ \\
\hline CFFLSD & $10 \%$ & $6.9 \%$ & $69.5 \%$ & $5.2 \% \quad 41.0 \%$ & $4.5 \%$ & $67.2 \%$ & $5.4 \% \quad 39.3 \%$ & $2.9 \%$ & $65.6 \%$ & $5.5 \% \quad 38.9 \%$ \\
\hline CFFLSD & $20 \%$ & $5.1 \%$ & $67.0 \%$ & $8.1 \% \quad 50.2 \%$ & $3.4 \%$ & $64.7 \%$ & $8.1 \% \quad 50.6 \%$ & $2.3 \%$ & $65.1 \%$ & $8.3 \% \quad 49.7 \%$ \\
\hline CFFLSD & $30 \%$ & $4.7 \%$ & $67.5 \%$ & $9.3 \% \quad 59.5 \%$ & $3.4 \%$ & $67.9 \%$ & $8.8 \% \quad 59.1 \%$ & $2.2 \%$ & $64.9 \%$ & $9.6 \% \quad 59.7 \%$ \\
\hline BFL & 0 & $4.1 \%$ & $3.5 \%$ & $\mathrm{~N} / \mathrm{A}$ & $2.9 \%$ & $2.9 \%$ & $\mathrm{~N} / \mathrm{A}$ & $1.9 \%$ & $2.5 \%$ & $\mathrm{~N} / \mathrm{A}$ \\
\hline BFL & $10 \%$ & $2.9 \%$ & $2.2 \%$ & $4.8 \% \quad 91.7 \%$ & $1.9 \%$ & $1.7 \%$ & $5.1 \% \quad 94.1 \%$ & $1.2 \%$ & $1.4 \%$ & $5.2 \% \quad 95.1 \%$ \\
\hline BFL & $20 \%$ & $2.0 \%$ & $1.3 \%$ & $13.7 \% \quad 95.0 \%$ & $1.3 \%$ & $1.0 \%$ & $14.4 \% \quad 96.7 \%$ & $0.9 \%$ & $0.7 \%$ & $14.4 \% \quad 96.7 \%$ \\
\hline BFL & $30 \%$ & $1.5 \%$ & $0.9 \%$ & $23.8 \% 96.2 \%$ & $1.0 \%$ & $0.7 \%$ & $24.4 \% \quad 97.3 \%$ & $0.6 \%$ & $0.5 \%$ & $25.2 \% \quad 97.8 \%$ \\
\hline
\end{tabular}



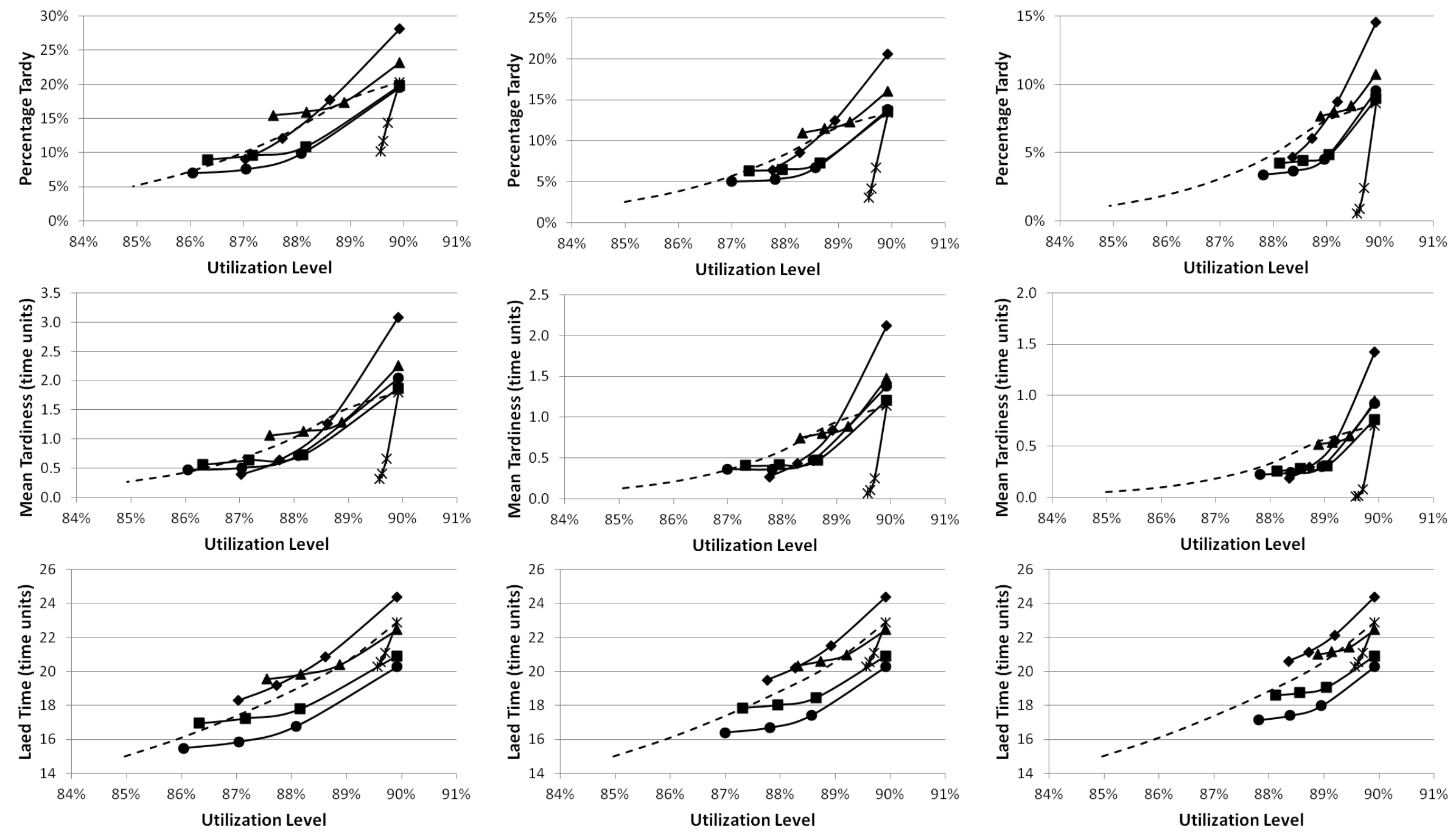

(a) Tight DD

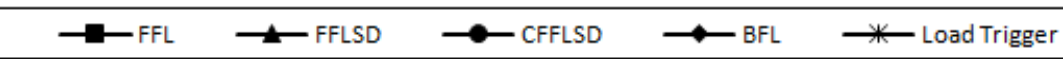

- - All adjusted

(b) Medium DD

(c) Loose DD

Figure 1: Performance Results for Different Levels of Due Date Tightness (Tight, Medium, and Loose Due Dates) with No Minimum Allowance 

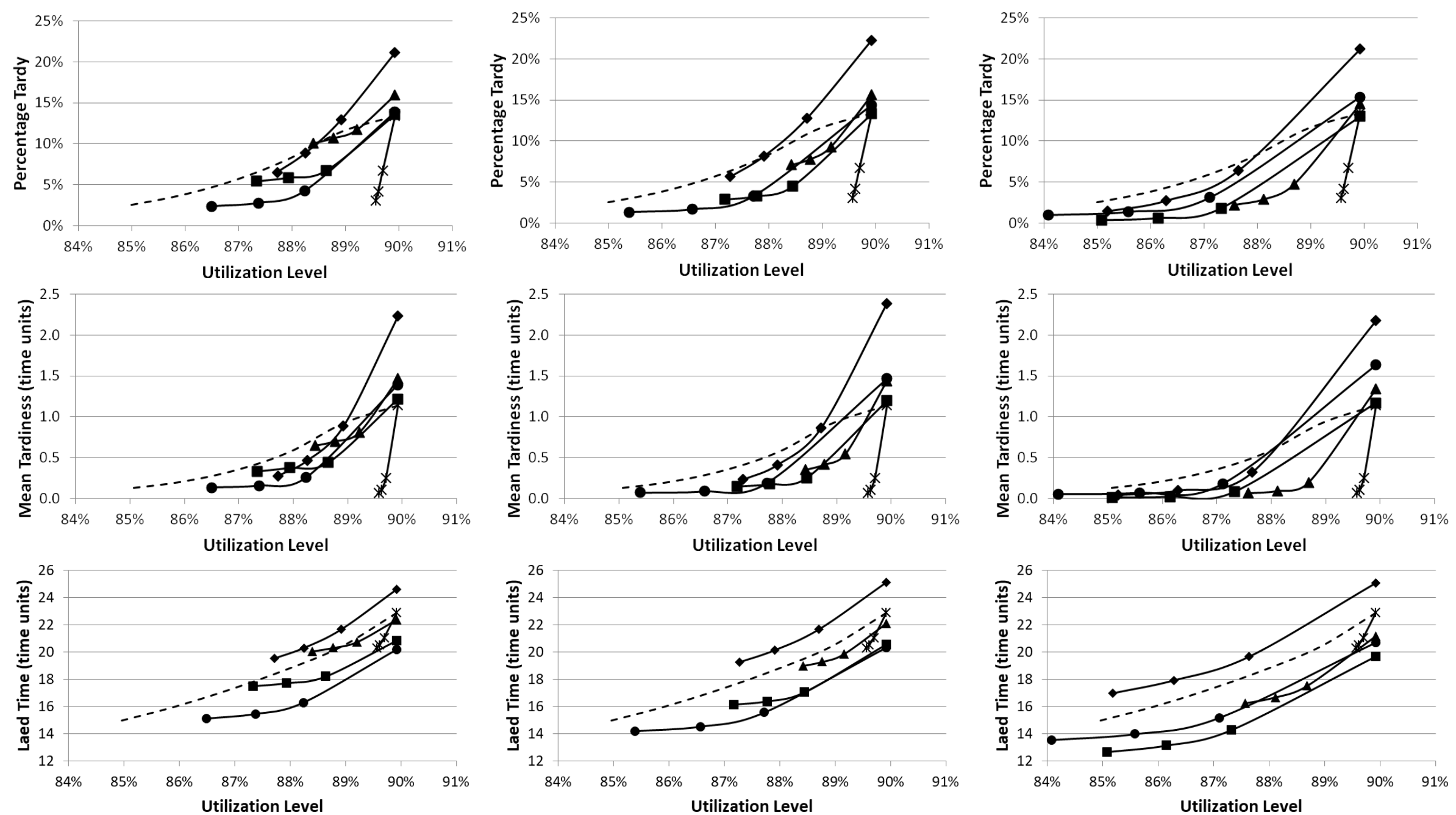

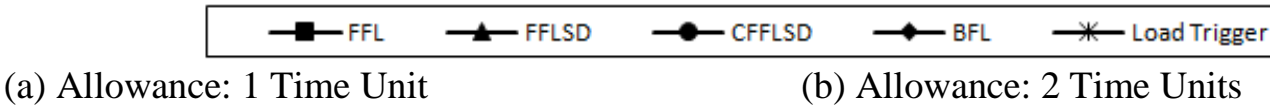

- - - All adjusted

(c) Allowance: 3 Time Units

Figure 2: Performance Results for Different Levels of the Minimum Allowance (1, 2, and 3 Time Units) at Medium Due Date Tightness 


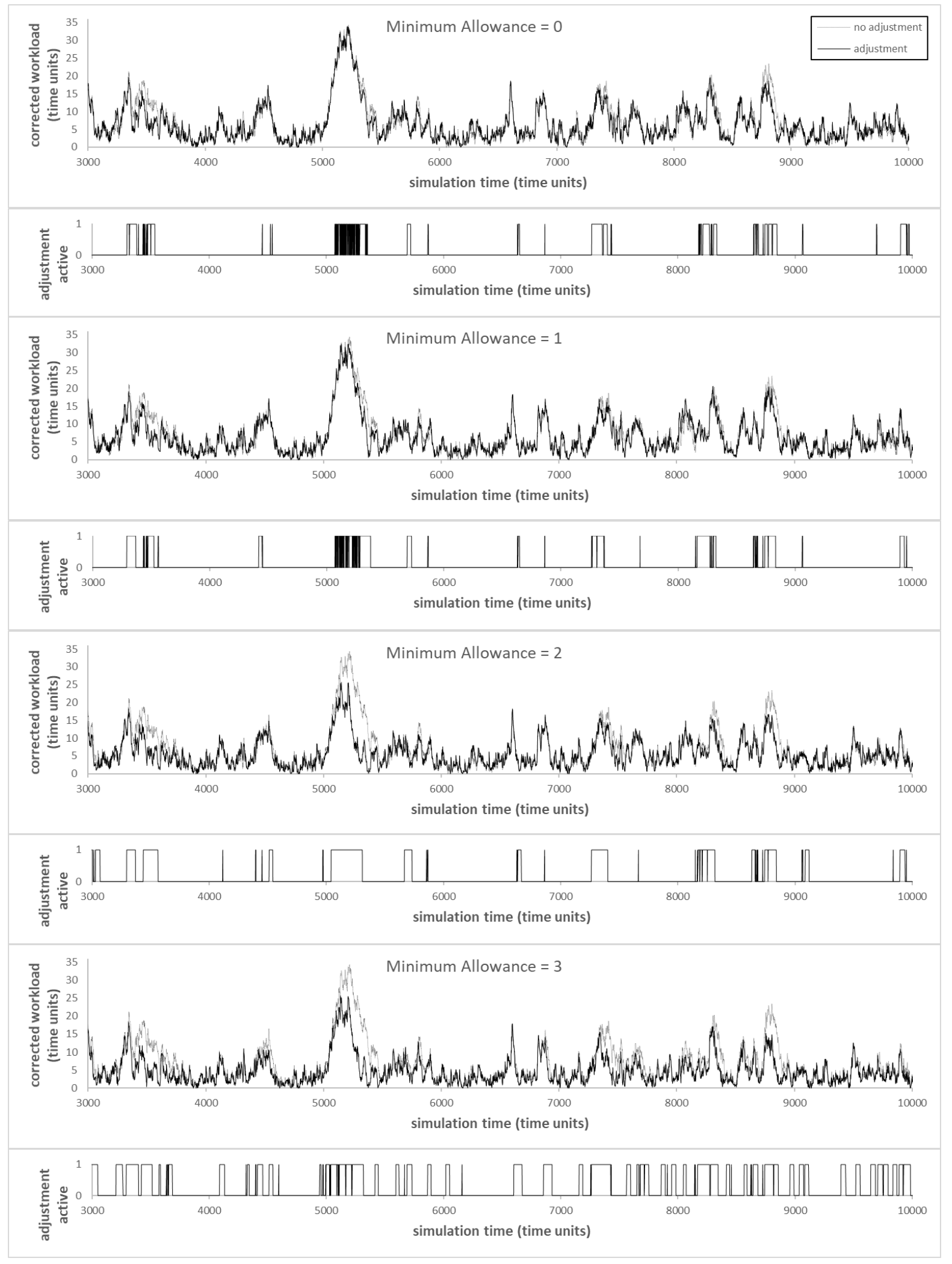

Figure 3: Workload Development under Different Minimum Allowances Over Time: FFL 


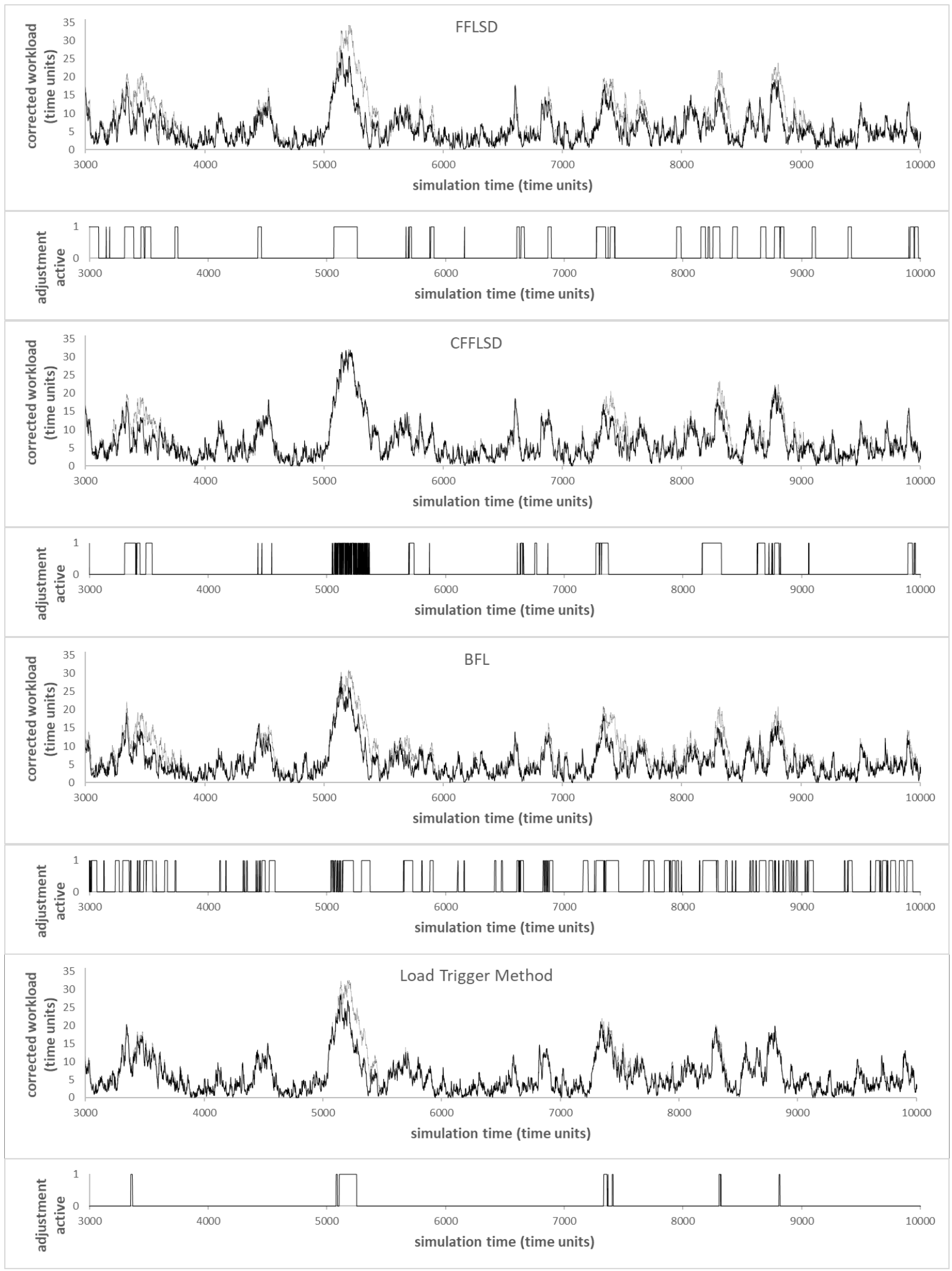

Figure 4: Workload Development for a Minimum Allowance of 3 Times Units: FFLSD, CFFLSD, and BFL 